• 研究报告・

\title{
一个种还是多个种？简化基因组及其形态学证据 揭示中国白岿椤植物的物种多样性分化
}

\author{
莫日根高娃 ${ }^{1,2}$ 商 辉 ${ }^{1}$ 刘保东 ${ }^{2}$ 康 明 ${ }^{3} \quad$ 严岳鸿 $^{1^{*}}$ \\ 1 (上海辰山植物园, 中国科学院上海辰山植物科学研究中心, 上海 201602) \\ 2 (哈尔滨师范大学黑龙江省普通高等学校植物生物学重点实验室, 哈尔滨 150025) \\ 3 (中国科学院华南植物园植物资源保护与可持续利用重点实验室, 广州 510650)
}

摘要: 物种是生物多样性的基本单元, 生殖隔离被认为是物种形成的关键; 然而物种并不是静止的而是处于不断 的分化演变之中, 已经稳定成型但尚未到达分化后期的物种可能存在不完全的生殖隔离。对于物种的认识不能单 从某一侧面或局部特征来界定，而应通过“整合物种概念”来确定物种地位。Flora of China记载了中国产白杪椤属 (Sphaeropteris) 2种, 即白杪椤 (S. brunoniana) 和笔筒树 (S. lepifera), 并认为原产中国海南的海南白杪椤 (S. hainanensis)和白杪椤为同一物种而将其并入白杪椤; 但海南白杪椤在形态上已出现了分化。为探讨白杪椤及其近 缘物种的亲缘关系和物种多样性分化的情况，本文采集到 9 个居群共 21 个样本，通过GBS简化基因组测序技术获 得单核苷酸变异位点(SNP), 进行系统发育树的构建和主成分及遗传结构的分析, 并结合叶片数量性状的统计分 析和孢子形态的观察测量。结果表明, 海南白杪椤不仅与云南产白杪椤的基因型不同, 且在叶片特征和孢子纹饰 上有明显差异; 但两个居群的生殖隔离较弱, 在广西沿海地区形成杂交产物, 其叶片特征为亲本的中间类型。因 此, 我们认为海南白杪椤是由于地理隔离而形成的一个处在分化路上的物种, 建议恢复其物种地位; 广西产白杪 椤为自然杂交群体，应另处理为独立的自然杂交分类群——广西白杪椤(S. brunoniana $\times$ hainanensis)。

关键词：白杪椤属; 海南白杪椤; 物种分化; 自然杂交; 基于测序的基因分型; 单核苷酸多态性

\section{One or more species? GBS sequencing and morphological traits evidence reveal species diversification of Sphaeropteris brunoniana in China}

\author{
Morigengaowa $^{1,2}$, Hui Shang ${ }^{1}$, Baodong Liu $^{2}$, Ming Kang ${ }^{3}$, Yuehong Yan ${ }^{1 *}$ \\ 1 Shanghai Chenshan Botanical Garden; Shanghai Chenshan Plant Science Research Center, Chinese Academy of \\ Sciences, Shanghai 201602 \\ 2 Key Laboratory of Plant Biology, College of Heilongjiang Province, Harbin Normal University, Harbin 150025 \\ 3 Key Laboratory of Plant Resources Conservation and Sustainable Utilization, South China Botanical Garden, Chinese \\ Academy of Sciences, Guangzhou 510650
}

\begin{abstract}
Species are not static, but in the process of continuous differentiation and evolution. In this context, reproductive isolation is considered the key to most speciation events. However, species that may be only partially reproductively isolated may disturb our understanding of a species, as stated by 'The integrative species concept'. In the Flora of China, Chinese Sphaeropteris contains two species, S. brunoniana and S. lepifera. S. brunoniana is thought to be the same species as a Hainan native, S. hainanensis, however, S. hainanensis seems to have differentiated morphologically. This study further explores the genetic relationship between $S$. brunoniana and its related species by collecting 21 samples from 9 populations for GBS reduced-representation genome sequencing for phylogenetic analysis. Using both genetic and morphological data, our results indicate that $S$. hainanensis differs genetically from $S$. brunoniana, but is also morphologically distinct based on leaf characteristics and spore ornamentation. Although the reproductive isolation of the two populations is weak, hybrids are formed in the coastal areas of Guangxi and leaves mimic
\end{abstract}

收稿日期: 2019-04-29; 接受日期: 2019-06-29

基金项目: 国家自然科学基金(31770350)、上海市绿化和市容管理局科技攻关项目(G182411)和湖南省教育厅科学研究项目(10C0720)

* 通讯作者 Author for correspondence. E-mail: yan.yh@126.com 
intermediate morphology between parents. Therefore, our results suggest that S. hainanensis is in the process of speciation due to geographic isolation and suggests to restore its species status. S. brunoniana $\times$ hainanensis which the sample from Guangxi should be considered an independent natural hybrid taxon.

Key words: Sphaeropteris; S. hainanensis; speciation; natural hybridization; GBS; SNP

物种是生物多样性的基本单元，但物种的边界 却难以确定。自林奈(1753)发表《植物种志》标志 着现代生物学的诞生以来, 生物界的物种被相继描 述; 但即使进入了基因组生物学时代, 关于水稻 (Oryza sativa)物种概念中的籼稻、粳稻命名问题仍 然是人们关注的热点(Wang et al, 2018)。不同领域的 生物学家的物种定义各不相同, 迄今为止形成了数 十个物种概念(洪德元, 2016)。与动物中相对严格的 生殖隔离不同, 自然杂交在植物中广泛发生, 以至 于很多植物学家都怀疑植物物种的存在, 认为它们 是人们任意构建的分类单元, 与代表生殖独立支系 或进化单元的离散的客观实体相反(Arnold, 1997)。 但是, Rieseberg等(2006)通过对超过400个属的植物 和动物的表现型和生殖特点进行分析的结果表明, 物种分类存在困难更多的可能是由于无性生殖、多 倍体和分类学家分类过细等原因造成, 植物物种比 动物物种更有可能代表生殖上具有独立性的种系。

毫无疑问, 与在动物界中一样, 物种在植物界 中同样是一个客观存在的独立实体, 但是如何判断 一个植物物种与其他物种之间的界限却比较困难。 洪德元(2016)认为一个科学的、可操作的物种概念 应该在形态上区别分明、在系统发生树上形成独立 的谱系并且有各自的生态位和独立的分布区。然而, 物种并不是静止的, 而是处于不断的分化演变之中, 自然界中存在大量处于分化路上的“物种”, 这些尚 未到达分化后期的物种可能存在不完全的生殖隔 离和有限的基因流(刘建全, 2016)。虽然现有的物种 概念“都揭示了物种属性的特定侧面”, 但都无法令 所有人满意(谢平, 2016); 这些学术观点的分歧可 能随着证据的增多而逐渐取得共识(孔宏智, 2016)。

随着分子生物学的发展, 尤其是近年来高通量 测序的发展, 大量的分子标记可以用于构建物种间 相对稳健、可信的亲缘关系; 同时随着标本采集量 的增加和电子显微镜技术的普遍应用, 大量的形态 学观察数据、超微结构变得更容易获取。这些数据 的综合应用使我们对 “整合物种概念”的认识成为可
能，而不至于如同过去仅仅从某一侧面或局部特征 定义物种，而导致在物种认识方面的“盲人摸象” (刘建全, 2016)。

蒌类植物是最早登陆的维管植物之一, 有 4 亿 多年的演化历史(李星学等, 1981; Morris et al, 2018)。其独特的生活史特征(Soltis \& Soltis, 1987) 致使复杂的多倍化和杂交频繁发生(Soltis \& Soltis, 1987; Rothfels et al, 2015); 配子体内自交(Klekowski \& Lloyd，1968)、同株植物配子体间自交 (Klekowski, 1969)和无融合生殖(Johri, 1984)等多种 生殖方式也频繁发生, 因此物种间的生殖隔离机制 并不完善, 物种间边界的界定也尤为困难。Flora of China记载中国杪椤科白杪椤属(Sphaeropteris)包括 白杪椤(S. brunoniana)、笔筒树(S. lepifera) 2种; 并 认为原产中国海南的海南白杪椤(S. hainanensis)和 白杪椤为同一物种而将其并入白杪椤 (Zhang \& Nishida, 2013)。实际上, Wang和Guan (2011)开展了 cpDNA的测序及种群遗传学分析, 发现海南白杪椤 与云南产的白杪椤之间存在遗传结构分化, 且云南 居群为原始单倍型。由于该研究没有开展核基因的 测序分析, 也没有开展更多的形态学方面的观察, 因此并未能全面地评估海南白杪椤物种的分类学 地位。本研究拟以中国白杪椤属为研究对象, 通过 GBS简化基因组测序技术获得单核苷酸变异位点 (single nucleotide polymorphism, SNP)对白杪椤属样 品进行遗传分析, 并结合形态学研究探讨白杪椤及 其近缘物种的亲缘关系和物种多样性分化情况。

\section{材料与方法}

\section{1 植物材料}

本研究在福建、广西、海南、台湾及云南地区 共计采集了 9 个居群 21 份白杪椤属植物的样本, 采 集点基本覆盖了该属植物在中国的分布地。每份样 品取3-5片小羽片用硅胶干燥以备后续DNA提取, 样品的具体采集信息见表1, 凭证标本存放于上海 辰山植物园标本馆 $(\mathrm{CSH})$ 。 
表1 白杪椤属的样品采集信息

Table 1 Sample details of Sphaeropteris

\begin{tabular}{lll}
\hline 类群 Taxon & 采样地点 Location & 样本数量及采集号 Sample size and voucher no. \\
\hline 白杪椤 S. brunoniana & 广西东兴 Dongxing, Guangxi & 3: CFH09001403, CFH09001405, CFH09001406 \\
白杪椤 S. brunoniana & 海南琼中 Qiongzhong, Hainan & 2: SG2100, SG2103 \\
白杪椤 S. brunoniana & 海南五指山 Wuzhishan, Hainan & 6: SG2005, SG2014, SG2021, SG2030, SG2050, SG2054 \\
白杪椤 S. brunoniana & 云南河口 Hekou, Yunnan & 3: CFH09000322, CFH09000324, CFH09000325 \\
白杪椤 S. brunoniana & 云南麻栗坡 Malipo, Yunnan & 1: YN384 \\
白杪椤 S. brunoniana & 云南屏边 Pingbian, Yunnan & 2: CFH09000301, ZXL05870 \\
笔筒树 S. lepifera & 福建福州 Fuzhou, Fujian & 1: GBJ02954 \\
笔筒树 S. lepifera & 福建平潭 Pingtan, Fujian & 1: GBJ04090 \\
笔筒树 S. lepifera & 台湾兰屿 Lanyu, Taiwan & 2: ZXL06026, ZXL09919 \\
\hline
\end{tabular}

\subsection{DNA提取和GBS测序}

每个样品取 $20 \mathrm{mg}$ 叶片研磨成粉末, 用新型植 物基因组DNA提取试剂盒(离心柱型, 天根公司)提 取植物总DNA，经浓度和纯度检测后的高质量 DNA用GBS方法(Sonah et al, 2013)进行建库和测序 (北京诺禾致源科技股份有限公司)。取 0.1-1 $\mu \mathrm{g}$ DNA用限制性内切酶(Mse I和Ecor I)进行酶切, 之 后在片段两端加接头, PCR扩增含有接头的tag序列, 将样品混合后通过电泳回收350-400 bp 区间的DNA 条带, 纯化后的产物在Illumina HiSeq测序平台上进 行双末端(paired-end) 150测序。

\section{3 序列比对及SNP的挖掘}

叶绿体序列以 GenBank 上的 Alsophila podophylla叶绿体全基因组(MG262389)为参考, 核序列 采用测序数据量最大的样本CFH09000325的组装 结果作为拟参考, 利用BWA比对软件( Li \& Durbin, 2009)将样品过滤后的有效数据(clean reads)与参考 基因组进行比对。采用SAMtools (Li et al, 2009)软件 进行群体SNP的检测, 用贝叶斯模型检测群体中的 多态性位点, 对获得的SNPs进行过滤。

\section{4 群体遗传分析}

\subsection{1 系统发育分析}

用PGDspider2 (Lischer \& Excoffier, 2012)将获 得的SNP数据转换为构建系统发育树的文件, 通过 生物进化分析网站CIPRES (Miller et al, 2010)提供 的jModelTest2 on XSEDE (Darriba et al, 2012)对 DNA 的最适替代模型进行评估, 用RAxML-HPC BlackBox (Stamatakis, 2014)构建最大似然树(ML), 用MRBAYES on XSEDE (Ronquist et al, 2012)构建
贝叶斯树 $(\mathrm{BI})$, 设置核苷酸替代类型数 $n s t ~=6$, 位点 间速率分布设置为rates = gamma, 以随机树为起始 树, 4条马尔科夫链(Monte Carlo Markov chains), 运行 $1,000,000$ 代，每100代取样 1 次，每次运算开始的前 2,500个样本作为老化值(bum-in)舍弃，运算停止时 “分裂频率平均标准差”降至0.01, 否则增加运算代 数(Bunawan et al, 2017), 并用SplitsTree 4 (Morrison, 2009)软件构建白杪椤属的系统发育网络。本文未选 取外类群，树根依据Dong和Zuo (2018)发表的杪椤 科系统位置来确定。

\subsection{2 主成分分析}

用GCTA (Yang et al, 2011)软件计算特征向量 以及特征值, 并应用 $\mathrm{R}$ 软件的biplot软件包绘制散 点图。

\subsection{3 群体遗传结构分析}

用Structure (Pritchard et al, 2000; Falush et al, 2003)软件对经过miss 0.4、maf 0.05过滤条件篎选后 得到的15,624 个位点进行聚类分析, 参数设置为: $\mathrm{K}=$ $1-7$, 每个K值重复 10 次运算, 运行代数为 $1,000,000$, 舍弃前500,000代，运行结束后使用Tracer v1.6进行 收敛性评估。使用线上Structure Harvester工具计算 最优 K值 (http://taylor0.biology.ucla.edu/struct_ harvest/), 然后用CLUMPP软件对重复运算结果进 行抽样分析(https://rosenberglab.stanford.edu/clumpp. html), 最终用R软件的ggplot2软件包绘制结果图。

\section{5 叶性状差异显著性分析}

用游标卡尺测量并统计样品的末回羽片长宽 比、羽裂深度、裂片叶脉对数和裂片对数等 4 个叶 性状的数据, 每个样本随机测量 10 次, 用 $\mathrm{R}$ 软件的 
agricolae软件包分析白杪椤属种间不同叶性状的差 异显著性，并用boxplot软件包绘制箱线图。

\section{6 孢子电镜扫描实验方法}

在JSZ 6型解剖镜下用昆虫针将孢子从样本中 挑取到粘有导电胶的铜台上, 每个样本挑取 30 个孢 子。将粘有样品的铜台置于LEICA EM ACE200型 喷金仪中喷金1 min, 后放入FEI QUANTA250型扫 描电子显微镜的样品台中, 并在 $30 \mathrm{kv}$ 下观察拍照。使 用Adobe Photoshop CC 2017的标尺工具测量狍子的 赤道轴和极轴的长度, 狍子纹饰描述主要参照文献 中所用术语(Ranker et al, 1993; 王全喜和于晶, 2003)。

\section{2 结果}

\section{1 白杪椤属系统发育树}

核基因系统发育树(图1A)基于18,319个SNP位 点, 运用贝叶斯法(BI)和最大似然法(ML)进行构 建。结果表明, 白杪椤属的 21 个样品聚为两支, 即 白杪椤和笔筒树, 支持率高达 PP $=1.0 、 \mathrm{BS}=100 \%$ 。 白杪椤支系分出两个亚支, 一个亚支的样品来自广 西和云南, 支持率为 $\mathrm{PP}=1.0 、 \mathrm{BS}=79 \%$, 而另一亚 支的样品均产自海南, 与另外两个产地的遗传距离 较远，支持率高达 $\mathrm{PP}=1.0 、 \mathrm{BS}=100 \%$ 。核基因的
网络系统发育树(图2)显示白杪椤按照3个产地聚为 3支, 且产自广西的白杪椤可能是海南和云南的杂 交种。

叶绿体基因系统发育树(图1B)是基于2,669个 SNP位点构建的，其拓扑结构与核基因系统发育树 基本一致, 由白杪椤和笔筒树两大支构成。但由于 SNP位点较少, 除了两大主支外, 其他亚分支的支 持率相对较低, 其中海南产白杪椤聚为 3 个亚支; 云南产白杪椤和广西产白杪椤共同聚为一支，表明 这两个产地的白杪椤在母系遗传基因上具有共同 的祖先。

\section{2 白杪椤属SNP位点的主成分分析}

主成分分析是基于18,319个核SNP位点进行的, 结果如图3所示。白杪椤属的 21 个样品聚为 4 个集群, 其中白杪椤分成了 3 个集群, 对应不同的样品采集 地, 即海南、广西和云南, 其中采自广西的样品与 云南的样品距离更近。

\section{3 白杪椤属的群体遗传结构}

Structure分析是基于15,624个核SNP位点进行 的聚类分析。依据Evanno等(2005)的统计方法得到 最大的 $\Delta \mathrm{K}$, 来确定最佳 $\mathrm{K}$ 值, 结果如图4所示。当 $\mathrm{K}$ $=3$ 时, $\Delta \mathrm{K}$ 出现一个明显的峰值, 说明此时白杪椤属

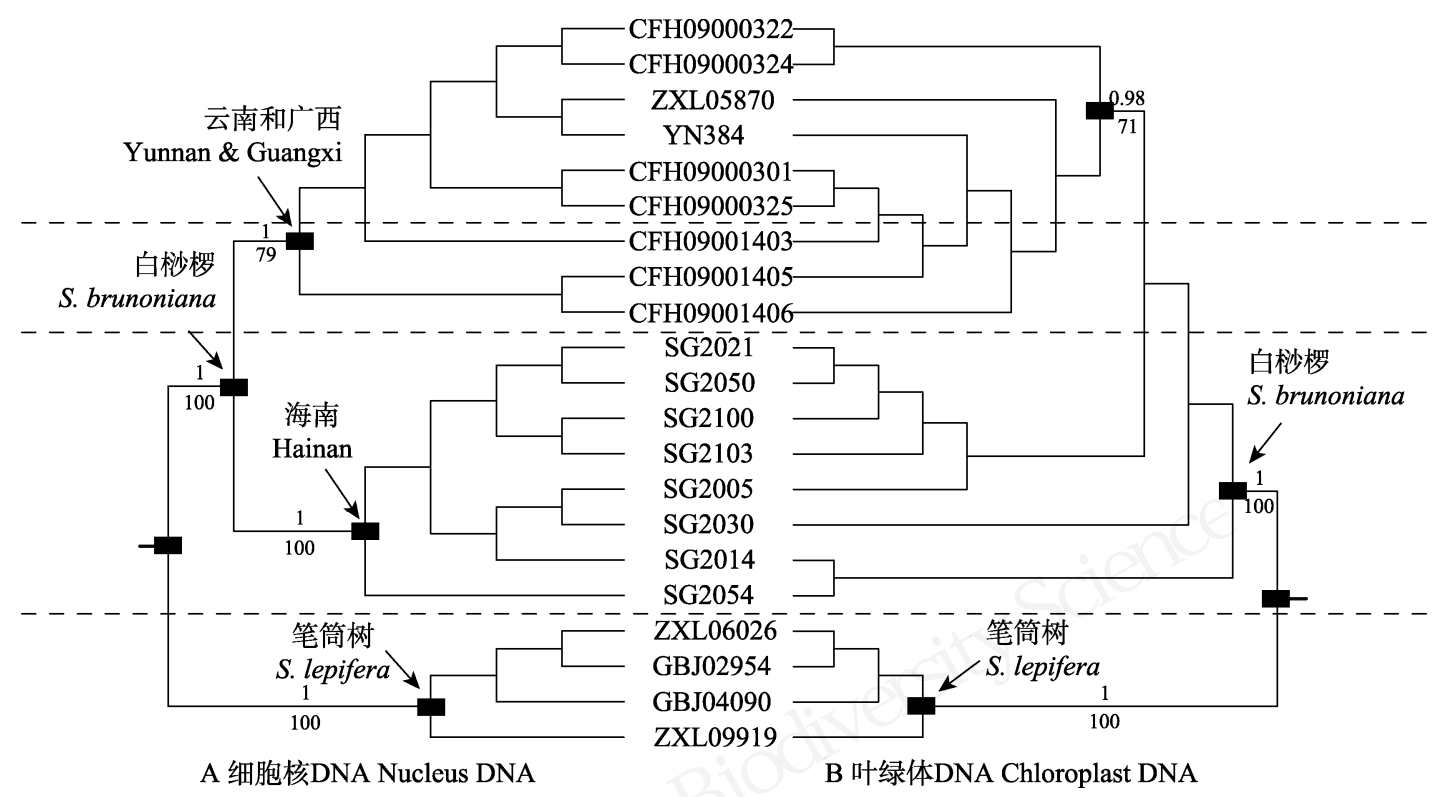

图1 运用贝叶斯法和最大似然法, 基于核基因和叶绿体基因构建的白杪椤属系统发育树。横线上方的数字表示贝叶斯后验 概率 $(P P \geq 0.95)$ ，横线下方的数字表示ML自展支持率 $(B S \geq 50 \%)$ 。样品信息见表 1 。

Fig. 1 The nuclear genes (A) and chloroplast genes (B) phylogenetic tree of Sphaeropteris based on Bayes and Maximum likelihood method. Numbers above the branches are posterior probability ( $\mathrm{PP} \geq 0.95$ ) from the BI analysis, and numbers under the branches are bootstrap percentages (BS $\geq 50 \%$ ) of maximum likelihood analysis. The sample information is shown in Table 1 . 


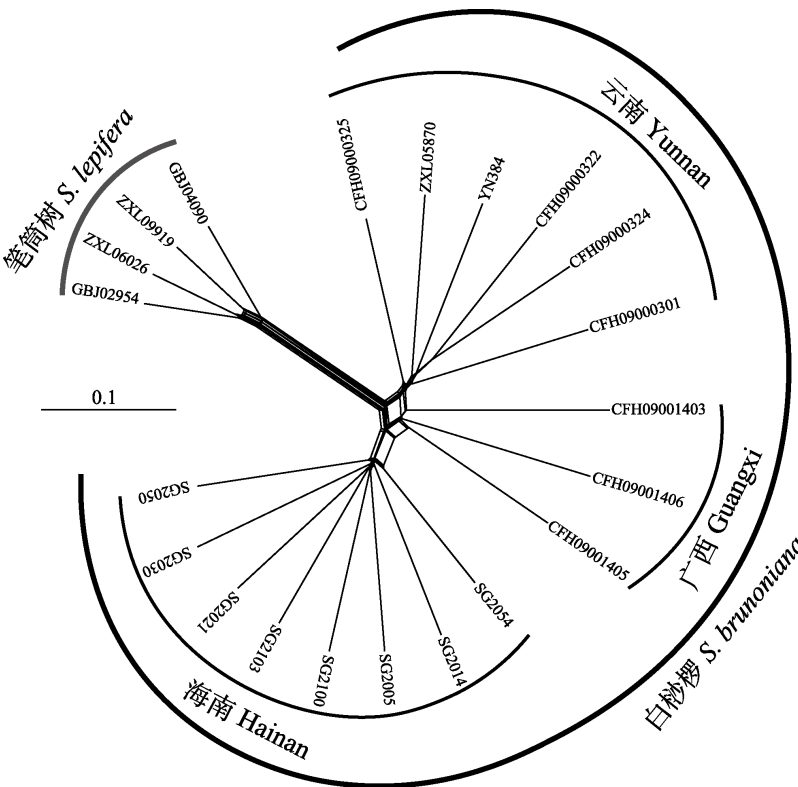

图2 基于核基因的SNP位点构建的白杪椤属邻接网络图 Fig. 2 Neighbor-Net network of Sphaeropteris based on nuclear SNPs

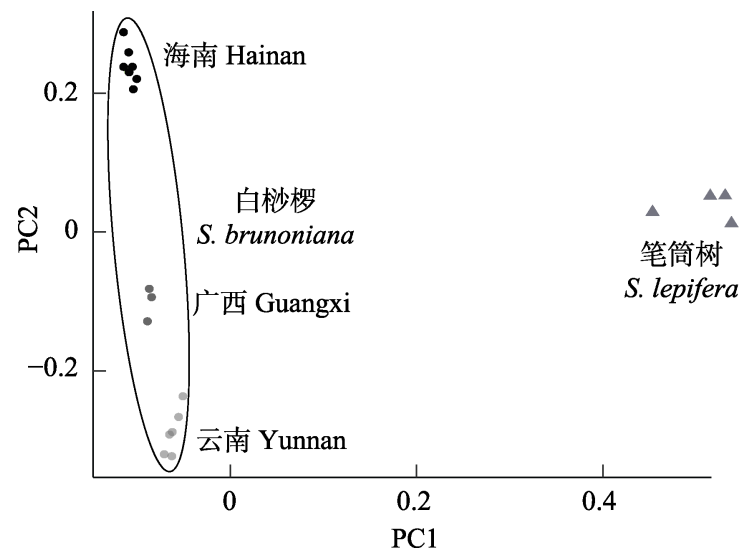

图3 白杪椤属基于SNP位点的主成分分析。图中每个点代 表1个样品。

Fig. 3 Principal component analysis of Sphaeropteris based on SNPs. Each sample is represented by a point.

群体内部符合哈迪-温伯格平衡, 因此这个临界点 的群体聚类结果最为真实可信。

$K=3$ 时的Structure结果如图5所示, 白杪椤 属分成3个集群, 即蓝色(海南产白杪椤)、绿色(云南 产白杪椤)和灰色(笔筒树)所代表的区域。采自海南 的白杪椤和采自云南的白杪椤样品具有完全不同 的基因型, 而来自广西的样品为另外两个产地白杪 椤的杂交产物，且其中来自云南的白杪椤的遗传物 质所占比例较大。

\section{4 白桫椤属种间羽片形态学差异}

将白杪椤样品按照产地分成3组, 与笔筒树共

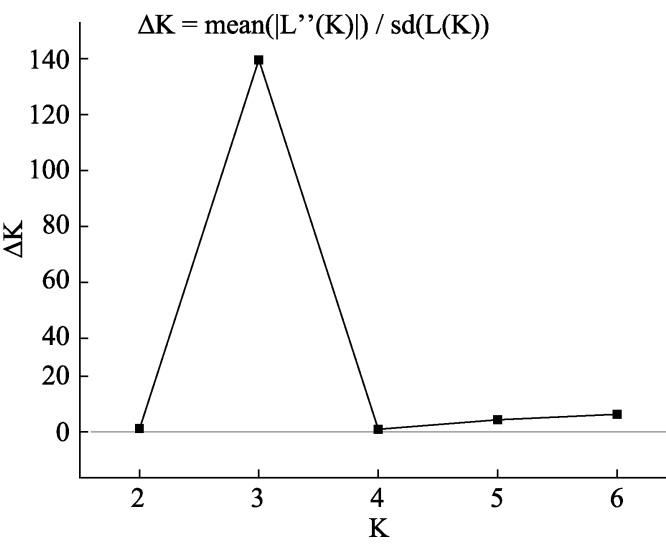

图4 通过 $\Delta K$ 的统计方法获得最优 $K$

Fig. 4 The optimal $\mathrm{K}$ is obtained by the statistical method of $\Delta \mathrm{K}$

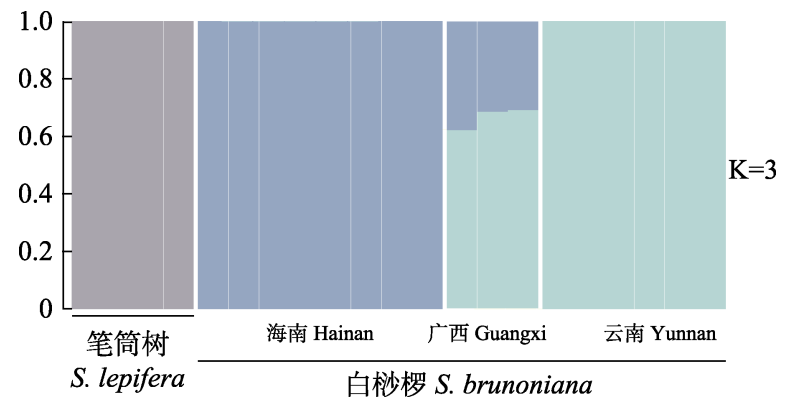

图5 白杪椤属Structure分析结果。每一个柱形代表1个样 本, 每种颜色代表 1 个遗传簇。

Fig. 5 Structure of Sphaeropteris. Each sample is represented by a histogram, which is partitioned into different colors. Each color represents a genetic cluster.

同进行叶片特征比较分析，结果如图6所示。笔筒树 和白杪椤两个物种在末回羽片长宽比和裂片对数 两个性状上有显著差异 $(P \leq 0.05)$, 而在裂片叶脉对 数和羽裂深度性状上与采自云南的白杪椤样品差 异较小。末回羽片长宽比: 云南产白杪椤末回羽片 平均长 $11.9 \mathrm{~cm}$ 、宽 $2.3 \mathrm{~cm}$, 长宽比值较大为 5.2 , 海南 产白杪椤末回羽片平均长 $9.1 \mathrm{~cm}$ 、宽 $2 \mathrm{~cm}$ ，长宽比值 较小, 为4.6, 广西产白杪椤末回羽片平均长 $10 \mathrm{~cm}$ 、 宽 $2 \mathrm{~cm}$ ，长宽比值居于二者之间，为5，稍接近云南 产样本。末回羽片裂片对数: 云南产样本裂片对数 最多, 达18-22对, 海南产样本裂片对数最少, 约 15-20对, 广西产样本裂片对数居于二者之间, 约 18-20对。裂片叶脉对数：云南产样本裂片上的小脉 对数最多, 约 9-10对, 海南产样本约8-10对, 广西 产样本居于二者之间，约9对，略靠近海南产白杪 椤样本。

\section{5 白桫椤属的种间孢子形态学差异}

如图7所示，白杪椤属狍子为四面型狍子，呈 
辐射对称, 具有三裂缝, 裂缝长度为孢子半径的 $2 / 3$ 或几达孢子的赤道轴, 孢子大小为(31-37) $\mu \mathrm{m} \times$ (45-47) $\mu \mathrm{m}$ ，每个物种的孢子具体特征见表2。本文 未采集到海南白杪椤的孢子, 图片(D 1-2)引自王全 喜和戴锡玲(2010)出版的著作, 与另外两个采集地 的白杪椤孢子作对比。三个产地的白杪椤孢子形状 相似, 但在孢子大小上: 云南样品比海南样品的极 轴短, 而赤道轴长, 广西的样品则居于二者中间, 其赤道轴长更接近云南样品; 在孢子周壁纹饰上, 三者均为颗粒状纹饰, 但云南样品的颗粒状纹饰分 布较疏, 而海南样品则较为密集, 广西样品的颗粒 纹饰则较大, 呈短棒状, 分布较密集。

\section{3 讨论}

本文的遗传分析结果均表明采自海南的白杪 椤样品与其他产地的样品遗传距离较远, 且 Structure结果显示海南和云南的白杪椤样品具有完全不
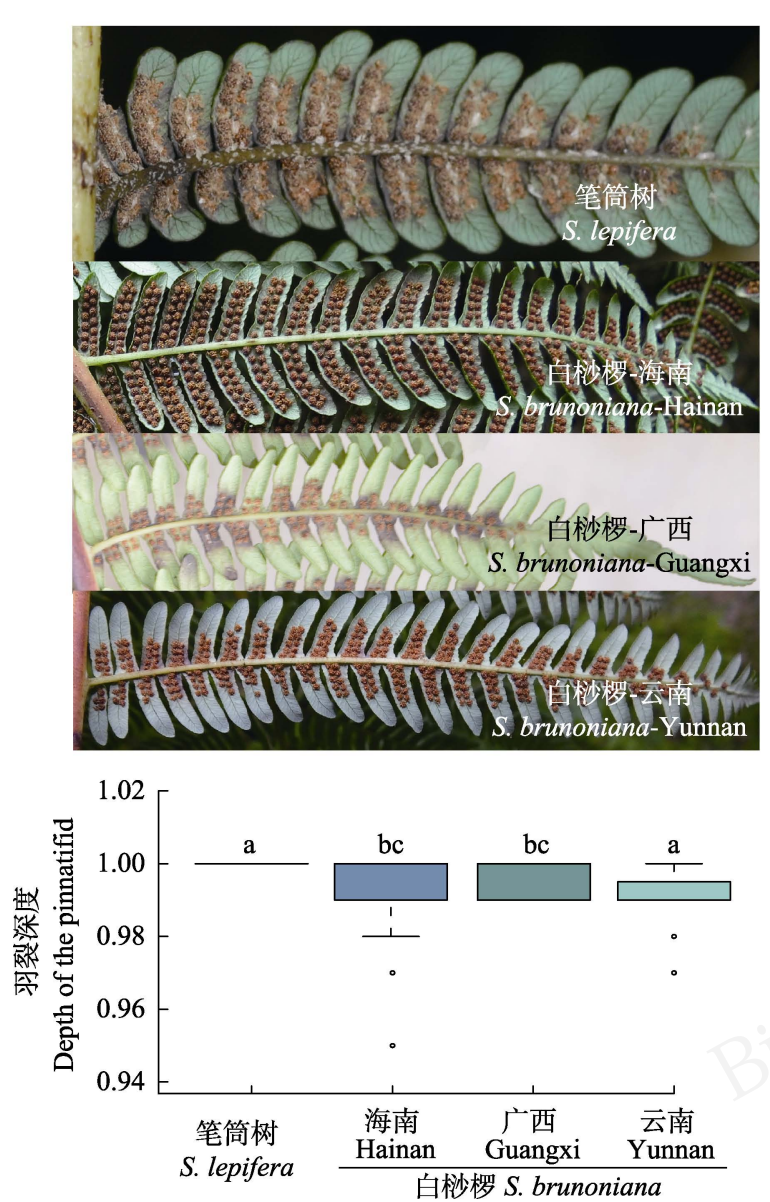

同的基因型，这与Wang和Guan (2011)的研究结果 一致, 她们发现海南产白杪椤具有与云南产白杪椤 完全不同的单倍型, 而云南的单倍型为祖先单倍型, 且海南与老挝的样品序列相似度极高, 她们认为海 南和云南的白桫椤是来自不同的谱系起源。Zhang 和Nishida (2013)认为海南白杪椤与白杪椤在形态 上没有区别, 所以将前者视为白杪椤的同一物种, 但本文在叶片特征的研究中发现, 海南和云南的样品 在形态上已经出现了分化, 两个居群在末回羽片长宽 比、裂片对数和裂片叶脉对数这三个叶性状上具有 显著性差异, 且孢子大小和纹饰也有明显的区别。

海南白杪椤和云南产白杪椤的生殖隔离较弱, 在云南和海南的中间区域即广西沿海地区形成了 杂交带(hybrid zone), 且整个广西地区只有这个杂 交带产白杪椤(广西壮族自治区中国科学院广西植 物研究所, 2013), 而位于杂交带上的白杪椤样品具 有海南和云南两种基因型, 且其样品的叶形态特征
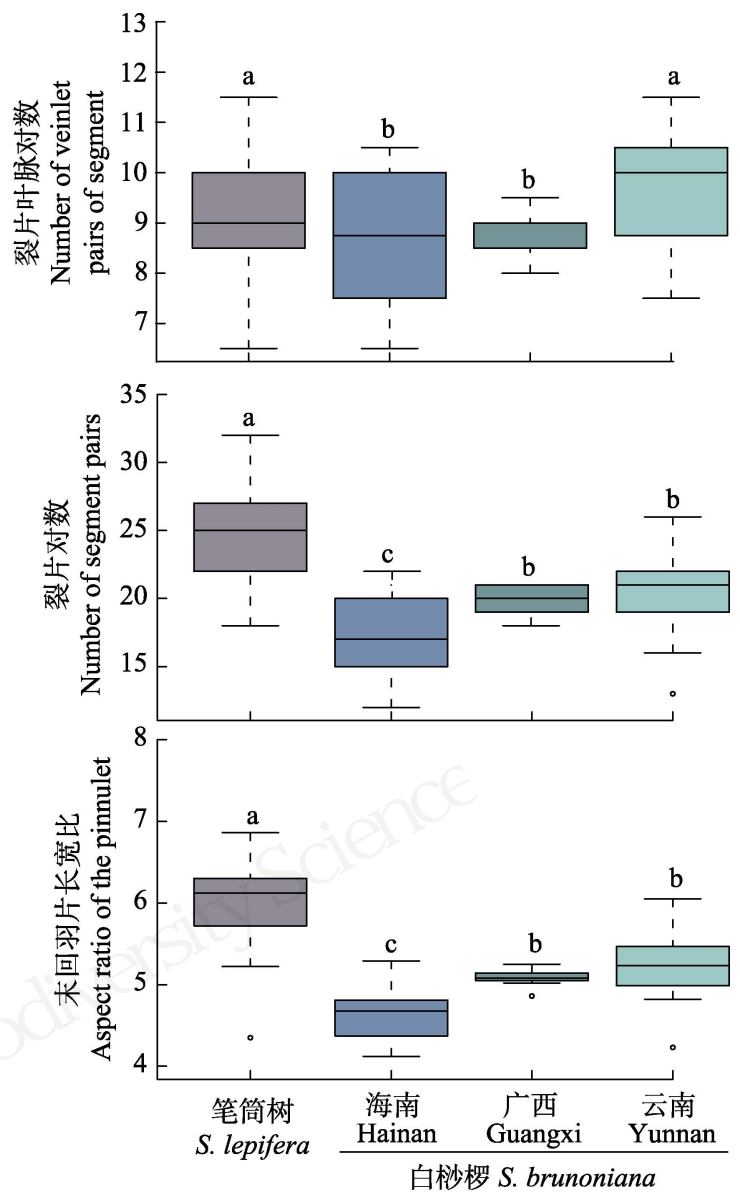

图6 白杪椤属种间叶性状差异显著分析

Fig. 6 The significant difference analysis in leaf traits of Sphaeropteris 

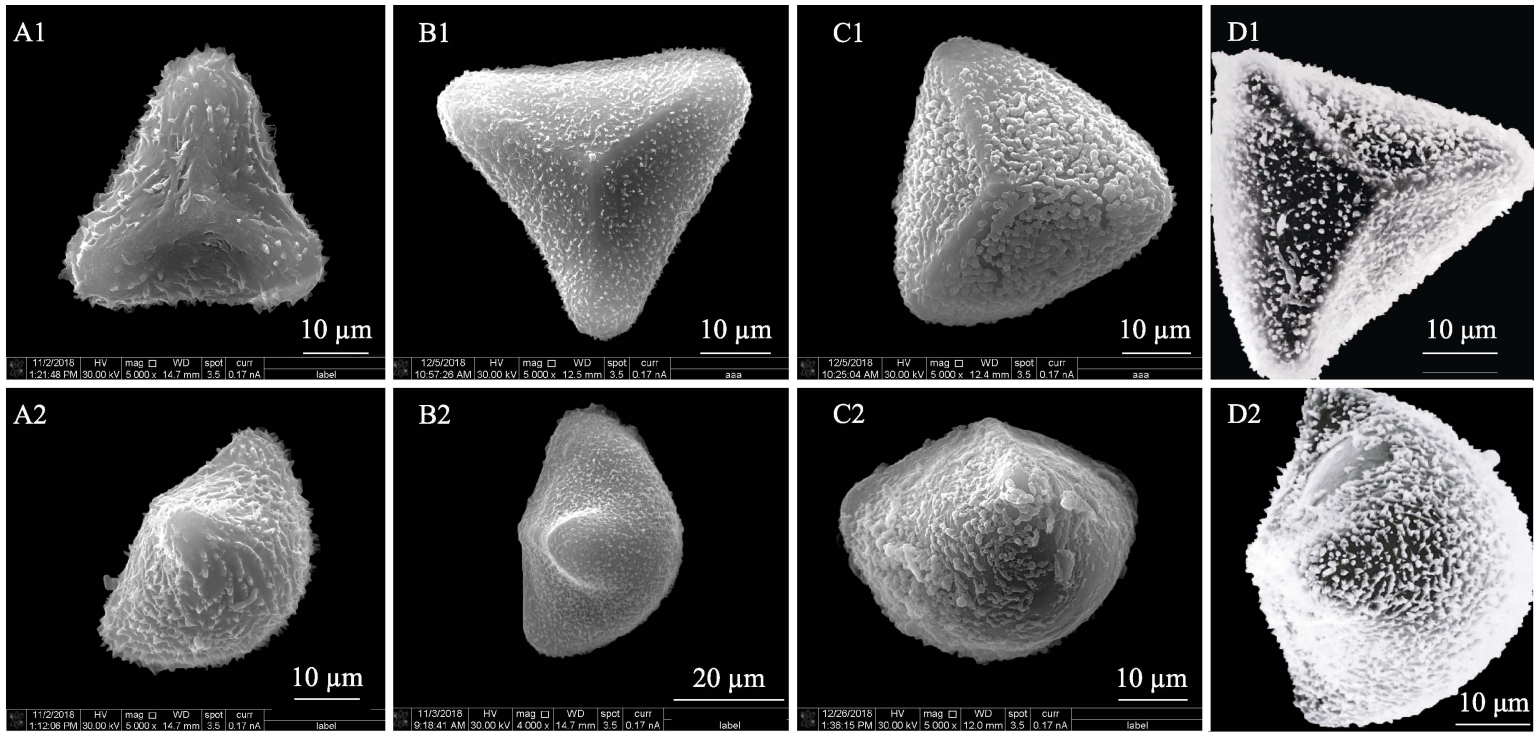

图7 白杪椤属孢子形态。A: 笔筒树; B-D: 白杪椤。B样品采自云南; C样品采自广西; D样品采自海南, 引自王全喜和戴锡 玲(2010)

Fig. 7 The spore morphology of Sphaeropteris. A, Sphaeropteris lepifera; B-D, Sphaeropteris brunoniana. B, Sample from Yunnan; C, Sample from Guangxi; D, Sample from Hainan, adopted fromWang \& Dai (2010).

表2 白杪椤属各种的孢子形态特征

Table 2 Spore morphological characteristics of Sphaeropteris

\begin{tabular}{lllll}
\hline & 笔筒树 & 白杪椤 S. brunoniana & & \\
\cline { 3 - 5 } & S. lepifera & 云南 Yunnan & 广西 Guangxi & 海南 Hainan \\
\hline 孢子大小 Spore size & $34 \mu \mathrm{m} \times 46 \mu \mathrm{m}$ & $31 \mu \mathrm{m} \times 47 \mu \mathrm{m}$ & $35 \mu \mathrm{m} \times 45 \mu \mathrm{m}$ & $37 \mu \mathrm{m} \times 45 \mu \mathrm{m}$ \\
极面观 & 三角形, 三边内凹 & 三角形, 三边内凹 & 三角形, 三边直 & 三角形, 三边直 \\
Polar view & Triangle, inset edge & Triangle, inset edge & Triangle, straight edge & Triangle, straight edge \\
赤道面观 & 扇形或近半圆形 & 扇形或近半圆形 & 扇形 & 扇形 \\
Equatorial view & Sector or approximate semicircle & Sector or approximate semicircle & Sector & Sector \\
表面纹 & 短刺状 & 颗粒状, 细小 & 颗粒状, 粗大 & 颗粒状, 细小 \\
Ornamentation & Short-echinulate & Granulate, small & Granulate, thick & Granulate, small \\
\hline
\end{tabular}

居于两个亲本的中间类型。广西产白杪椤在叶绿体 基因系统发育树上与云南产白杪椤聚在一起，故而 认为云南产白杪椤是其母本, 但有趣的是在核基因 系统发育树上其仍与云南的样品聚在一起, 且 Structure 结果显示云南产白杪椤的遗传物质所占比 重较大, 因此我们推测, 海南白杪椤是其父本, 但 杂交后代又与云南产白杪椤发生数次回交, 致使广 西产白杪椤与母本的遗传距离更近, 这与生态地理 距离相符。

尽管生殖隔离被认为是物种形成的关键和保 证物种独立性的基础(Coyne \& Orr, 2004), 但刘建 全(2016)认为物种始终处于 “分化路上”, 或在分化 的早期, 或处于中期, 亦或是后期。当物种处于分 化的早中期时, 其特征可能只满足其中一个或两个 物种概念的条件, 可能不具备完全的生殖隔离; 当
处于分化后期时, 才能够满足更多甚至所有物种概 念的条件, 而在自然界中处于分化后期的物种相对 较少。对于物种的认识, 我们不能单从某一侧面或 局部特征来界定，而应通过“整合物种概念”来确定 物种地位。地理隔离造就了海南白杪椤的遗传分化, 其与云南产白杪椤在形态上也存在差异, 但二者可 以交配形成同倍可育的子代。由此可见，尽管杪椤 科植物起源古老(Tryon et al, 1982), 但海南白杪椤 可能是新近才开始分化的年轻物种，尽管其仍处于 物种分化的路上，但本文建议恢复海南白杪椤的物 种地位，从而提高人们对该物种的保护意识。鉴于 此，中国产白杪椤属植物种类至少可区分为笔筒 树、白杪椤、海南白杪椤及后两者的自然杂交群体 广西白杪椤 $($ S. brunoniana $\times$ hainanensis), 各分类群 特征检索如下: 
1. 叶轴及羽轴密被疮突; 主脉下面被卵状平展的鳞片和灰白色长毛; 孢子周壁表面凹凸不平, 纹饰呈短刺 状. ..1. 笔筒树 S. lepifera (Hook.) R. M. Tryon

1. 叶轴及羽轴光滑; 主脉下面通常光滑或略被黄白色毛; 孢子周壁表面粗糙, 纹饰呈颗粒状.

2. 孢子表面颗粒较细小.

3. 末回羽片长约 $9-13 \mathrm{~cm}$, 裂片对数约18-22对; 孢子极轴长约 $29-31 \mu \mathrm{m}$.

2. 白杪椤 S. brunoniana (Hook.) R. M. Tryon

3. 末回羽片长约 $7-10 \mathrm{~cm}$, 裂片对数约 15-20对; 孢子极轴长约 $37 \mu \mathrm{m}$.

\section{..3. 海南白杪椤 S. hainanensis (Ching) R. M. Tryon}

2. 孢子表面颗粒较粗大 ..4. 广西白杪椤 S. brunoniana $\times$ hainanensis hybrid nov.

致谢: 感谢葛斌杰助理研究员、陈涁博士、顾钰峰、 罗俊杰、沈槂、韦宏金、张九兵及周喜乐对野外调 查和样品采集的帮助。

\section{参考文献}

Arnold ML (1997) Natural Hybridization and Evolution. Oxford University Press, Oxford.

Bunawan H, Yen CC, Yaakop S, Noor NM (2017) Phylogenetic inferences of Nepenthes species in Peninsular Malaysia revealed by chloroplast (trnL intron) and nuclear (ITS) DNA sequences. BMC Research Notes, 10, 67.

Coyne JA, Orr HA (2004) Speciation. Sinauer Associates, Sunderland, MA.

Darriba D, Taboada GL, Doallo R, Posada D (2012) jModelTest 2: More models, new heuristics and parallel computing. Nature Methods, 9, 772.

Dong SY, Zuo ZY (2018) On the recognition of Gymnosphaera as a distinct genus in Cyatheaceae. Annals of the Missouri Botanical Garden, 103, 1-23.

Evanno G, Regnaut S, Goudet J (2005) Detecting the number of clusters of individuals using the software STRUCTURE: A simulation study. Molecular Ecology, 14, 2611-2620.

Falush D, Stephens M, Pritchard JK (2003) Inference of population structure using multilocus genotype data: Linked loci and correlated allele frequencies. Genetics, 164, 1567-1587.

Guangxi Institute of Botany, Guangxi Zhuang Autonomous Region and the Chinese Academy of Sciences (2013) Flora of Guangxi, Vol. 6. Guangxi Science and Technology Publishing House, Xining. (in Chinese) [广西壮族自治区中国科 学院广西植物研究所 (2013) 广西植物志, 第6卷. 广西 科学技术出版社, 西宁.]

Hong DY (2016) Biodiversity pursuits need a scientific and operative species concept. Biodiversity Science, 24, 979-999. (in Chinese with English abstract) [洪德元 (2016) 生物多 样性事业需要科学、可操作的物种概念. 生物多样性, 24, 979-999.]

Johri BM (1984) Embryology of Angiosperms. Springer-Verlag, Berlin.
Klekowski EJ (1969) Reproductive biology of the Pteridophyta. II. Theoretical considerations. Botanical Journal of the Linnean Society, 62, 347-359.

Klekowski EJ, Lloyd RM (1968) Reproductive biology of the Pteridophyta. I. General considerations and a study of Onoclea sensibilis L. Botanical Journal of the Linnean Society, 60, 315-324.

Kong HZ (2016) Biodiversity undertakings call for extensive discussion on species concept and the criteria for species delimitation. Biodiversity Science, 24, 977-978. (in Chinese) [孔宏智 (2016) 生物多样性事业呼唤对物种概念和 物种划分标准的深度讨论. 生物多样性, 24, 977-978.]

Li H, Durbin R (2009) Fast and accurate short read alignment with Burrows-Wheeler transform. Bioinformatics, 25, 1754-1760.

Li H, Handsaker B, Wysoker A, Fennell T, Ruan J, Homer N, Marth G, Abecasis G, Durbin R (2009) The Sequence Alignment/Map format and SAMtools. Bioinformatics, 25, 2078-2079.

Li XX, Zhou ZY, Guo SX (1981) The Development and Evolution of the Plant Kingdom. Science Press, Beijing. (in Chinese) [李星学, 周志炎, 郭双兴 (1981) 植物界的发展 和演化. 科学出版社, 北京.]

Lischer HEL, Excoffier L (2012) PGDSpider: An automated data conversion tool for connecting population genetics and genomics programs. Bioinformatics, 28, 298-299.

Liu JQ (2016) “The integrative species concept” and "species on the speciation way”. Biodiversity Science, 24, 1004-1008. (in Chinese with English abstract) [刘建全 (2016) “整合物 种概念” 和 “分化路上的物种”. 生物多样性, 24, 10041008.]

Miller MA, Pfeiffer W, Schwartz T (2010) Creating the CIPRES science gateway for inference of large phylogenetic trees. In: Proceedings of the Gateway Computing Environments Workshop (GCE), New Orleans.

Morrison DA (2009) Using data-display networks for exploratory data analysis in phylogenetic studies. Molecular Biology Evolution, 27, 1044-1057.

Morris JL, Puttick MN, Clark JW, Edwards D, Kenrick P, 
Pressel S, Wellman CH, Yang ZH, Schneider H, Donoghue PCJ (2018) The timescale of early land plant evolution. Proceedings of the National Academy of Sciences, USA, 115, 2274-2283.

Pritchard JK, Stephens M, Donnelly P (2000) Inference of population structure using multilocus genotype data. Genetics, 155, 945-959.

Ranker TA, Tyron AF, Lugardon B (1993) Spores of the Pteridophyta: Surface, wall structure, and diversity based on electron microscope studies. Systematic Botany, 18, 377-378.

Rieseberg LH, Wood TE, Baack EJ (2006) The nature of plant species. Nature, 440, 524-527.

Ronquist F, Teslenko M, van der Mark P, Ayres DL, Darling A, Höhna S, Larget B, Liu L, Suchard MA, Huelsenbeck JP (2012) MrBayes 3.2: Efficient Bayesian phylogenetic inference and model choice across a large model space. Software for Systematics and Evolution, 61, 539-542.

Rothfels CJ, Johnson AK, Hovenkamp PH, Swofford DL, Roskam HC, Fraser-Jenkins CR, Windham MD, Pryer KM (2015) Natural hybridization between genera that diverged from each other approximately 60 million years ago. The American Naturalist, 185, 433-442.

Soltis DE, Soltis PS (1987) Polyploidy and breeding systems in homosporous pteridophyta: A reevaluation. The American Naturalist, 130, 219-232.

Sonah H, Bastien M, Iquira E, Tardivel A, Légaré G, Boyle B, Normandeau É, Laroche J, Larose S, Jean M, Belzile F (2013) An improved genotyping by sequencing (GBS) approach offering increased versatility and efficiency of SNP discovery and genotyping. PLoS ONE, 8, e54603.

Stamatakis A (2014) RAxML version 8: A tool for phylogenetic analysis and post-analysis of large phylogenies. Bioinformatics, 30, 1312-1313.

Tryon RM, Tryon AF, Rudolph ED (1982) Ferns and allied plants with special reference to tropical America. American Fern Journal, 73(3), 94-95.

Wang QX, Dai XL (2010) Spores of Polypodiales (Filicales) from China. Science Press, Beijing. (in Chinese) [王全喜, 戴锡玲 (2010) 中国水龙骨目(真䓲目)植物狍子形态的研
究. 科学出版社, 北京.]

Wang QX, Yu J (2003) Classification of spore ornamentation in filicales under SEM. Acta Botanica Yunnanica, 25, 313-320. (in Chinese with English abstract) [王全喜, 于晶 (2003) 扫描电镜下真蕨目孢子表面纹饰的分类. 云南植 物研究, 25, 313-320.]

Wang WS, Mauleon R, Hu ZQ, Chebotarov D, Tai SS, Wu ZC, Li M, Zheng TQ, Fuentes RR, Zhang F, Mansueto L, Copetti D, Sanciangco M, Palis KC, Xu JL, Sun C, Fu BY, Zhang HL, Gao YM, Zhao XQ, Shen F, Cui X, Yu H, Li ZC, Chen ML, Detras J, Zhou YL, Zhang XY, Zhao Y, Kudrna D, Wang CC, Li R, Jia B, Lu JY, He XC, Dong ZT, Xu JB, Li YH, Wang M, Shi JX, Li J, Zhang DB, Lee SH, Hu WS, Poliakov A, Dubchak I, Ulat VJ, Borja FN, Mendoza JR, Ali J, Li J, Gao Q, Niu YC, Yue Z, Naredo MEB, Talag J, Wang XQ, Li JJ, Fang XD, Yin Y, Glaszmann JC, Zhang JW, Li JY, Hamilton RS, Wing RA, Ruan J, Zhang GY, Wei CC, Alexandrov N, McNally KL, Li ZK, Leung H (2018) Genomic variation in 3,010 diverse accessions of Asian cultivated rice. Nature, 557, 43-49.

Wang ZJ, Guan KY (2011) Genetic structure and phylogeography of a relict tree fern, Sphaeropteris brunoniana (Cyatheaceae) from China and Laos inferred from cpDNA sequence variations: Implications for conservation. Journal of Systematics and Evolution, 49, 72-79.

Xie P (2016) A brief review on the historical changes in the concept of species. Biodiversity Science, 24, 1014-1019. (in Chinese with English abstract) [谢平 (2016) 浅析物种概 念的演变历史. 生物多样性, 24, 1014-1019.]

Yang J, Lee SH, Goddard ME, Visscher PM (2011) GCTA: A tool for genome-wide complex trait analysis. American Journal of Human Genetics, 88, 76-82.

Zhang XC, Nishida H (2013) Cyatheaceae. In: Flora of China, vol. 2-3 (Pteridophytes) (eds Wu ZY, Raven PH, Hong DY), pp. 134-138. Science Press, Beijing \& Missouri Botanical Garden, St. Louis.

(责任编委: 郭亚龙 责任编辑: 问文杰) 